\title{
Towards a New Science of Information
}

\author{
Dail Dewitt Doucette
}

daildd@comcast.net, Science of Information Institute, Washington, DC USA

\begin{abstract}
This paper suggests a New Information Paradigm and recommends the establishment of a new academic and scientific discipline to be titled "Science of Information", built on a premise that information is also a major part of all the universes, elements, systems, and conditions. Therefore, it is also an integral part of all the other individual disciplines and sciences. It is essential to look beyond the limitations of how humans use and perceive information, or even how other living organisms use information. It is proposed that information is a continuous evolving process, that exists in some simple to complex form in every stage of development, across all scientific and academic domains, as well as being a significant part of everything that exists. Information is a trigger mechanism, emphasis and nutrient, not only for information activities itself but also all physical, and biological elements, systems and activities. Each existing discipline has within it a significant core Informational Element, which helps formulate and define that discipline. The Science of Information will bring together these core information elements in order to correlate, compare and assemble a combined theoretical base. This new discipline should be established as a meta-discipline. It should exist equally with traditional discipline categories such as physical, social science, arts, and humanities. It should not compete with or replace other disciplines. It should stand alongside them and assist them, as well as conduct comparisons and consolidations amongst multiple disciplines regarding information theories, methodologies, practices, by adding new perspectives, resources and developments.
\end{abstract}

Keywords: Science of Information, Physical Sciences, Information Science, Transdisciplinary, Meta discipline, and New Academic Discipline

Acknowledgement: I want to thank Professor Kang Oyang and Professor E. Zin Zhong for hosting this important fourth FIS conference on the Foundations of Information Science, and Professor Zong-Rong Li for all his work in making the conference a success. The Science of Information Institute is very pleased to be a co-sponsor. As the Executive Director of the Institute, I can say on behalf of our Board of Directors and Scientific Advisory Committee - headed by Wolfgang Hofkirchner - that we look forward to continued productive discussions and concrete outcomes. I appreciate that so many young people have joined us at this conference. We dedicate our work to them and their future. The Science of Information is a Science of and for this new age.

The purpose of this paper is to challenge the active practitioners and participants in the field of information foundational and application studies, rather than being a standard academic paper. The observations and suggestions presented here are my own, and do not necessarily represent any organization. My duty here is to present some candid observations and suggestions to those actively involved in developing a new science of information.

\section{Proposed Perspective}

I suggest that all of you here attending this conference, members of Foundations of Information Science (FIS), Science of Information Institute (Scil), our Chinese hosts, and the Huangzhong University of Science and Technology, and other distinguished professional scholars from other countries, consider establishing a new information paradigm, together we would change our focus and activities, from the traditional studies in information science, semiotics, computer science, artificial intelligence, social information sciences, informatics, etc. into a new broader meta-discipline and professional domain, to be called a "Science of Information." The focus of this new field will be to assist in redirecting societies' current old hard, material centered culture, towards a newer softer, more responsive, and adaptable information centered culture. We must recognize, respect, and even support those traditional disciplines that helped us originate this evolved discipline to this point, but we must now focus our main attention on establishing this newer macro field, and assist in bringing it to reality. 
At the third FIS conference on Foundations of Information Science in Paris, 2005, the need for a science of information was recognized. We added an additional day to the conference to discuss this topic; out of this meeting we initiated the Science of Information Institute. This new international activity was housed in Washington, D.C., not because it represented any U.S. interests, but because that was where a support facility was initially offered. In November of 2005, at the 2nd UNESCO Conference on The Information Society in Tunis, Tunisia, I distributed a paper entitled "Information as a Unique and Scholarly Field" in which a Science of Information concept was presented to the United Nations and the international education and scientific communities. In both papers it was suggested that we have evolved beyond the traditional study of Information Theory and - Information Science, and should consider a new term "Science of Information" in order to broaden our scope and include the extended theories and perspectives of the physical and biological sciences, together with arts and humanities.

I now propose that the developments in the field are at a stage where "Science of Information" should be considered more a "meta-science" that is contributive to - and complementary with every other science and academic discipline rather than just a solitary traditional discipline that is normally distinguished by a single scientific perspective. Historically, studies of Information Science have included the fields of library and computer, science, semiotics, psychology and many social sciences, plus management and engineering-oriented approaches; all have been oriented toward the descriptive and manipulative aspects of information.

Information seems to have four interacting properties of information that we need to explore more fully.

A. It is descriptive (humans most often think of information in this mode)

B. It has manipulative, computative, relativeness capabilities (mathematics, computer science are more aware of this aspect of information)

C. It has prescriptive directive, capabilities (physics, cosmology, managers, and motivators are more aware of this property of information)

D. It performs these properties upon informational energy and elements as well as physical energy and elements

These properties seem to be somewhat common across the informational world of physical, social and applied sciences.

The concepts presented by Erwin Schrödinger in his publication "What is Life, with Mind and Matter" (1992), and Carl Fredrich von Weizsäcker, while at the Max Planck Institute, plus physicists John Wheeler, Anton Zeilinger, Hans Christian von Baeyer, and philosopher Luciano Floridi all have shown how information in physical sciences additionally includes a "prescriptive" element that initiates and contribute to the development of information. In a similar manner, in addition to the more traditional descriptive and manipulative perspectives, the science of information must also now have a strong inclusion of the physical and biological science approaches. In sociology, there is a distinction between the smaller nuclear family and the larger extended family. What is proposed here is that the study of information follows this analogy of an extended family, and includes all the other disciplines' studies and developments as contributive elements of information studies - all under one science of information umbrella.

\section{The Current Structure of Academia and Disciplines of Science}

If we consider the Science of Information as an evolving field of study and development, a question for those interested in this field is, how to place or categorize information as a unique field of study, within the current academic and science disciplines.

Although Wikipedia is not the most authoritative reference on the subject, it is easier to use, and more indicative of a contemporary broad perspective than the detailed UNESCO classification of 
sciences. The following is a summary list from Wikipedia of 58 standard science and academic disciplines, grouped in 8 standard academic/science clusters.

Science Specializations:

1. Natural/Physical Sciences (some cultures refer to this as material sciences)
1.1. Astronomy
1.2. Chemistry
1.3. Cosmology
1.4. Earth Sciences
1.5. Environmental Science
1.6. Physics

2. Formal Sciences

2.1. Computer Science

2.2. Logic

2.3. Mathematics

2.4. Statistics

2.5. System Sciences

3. Natural Life Sciences

3.1. Anatomy

3.2. Biology

3.3. Botany

3.4. Ecology

3.5. Evolution

3.6. Genetics

3.7. Neurology

3.8. Physiology

3.9. Zoology

4. Cognitive Sciences

4.1. Communication and Journalism

4.2. Informatics

4.3. Information Science

4.4. Library Science

4.5. Linguistics

4.6. Neuroscience

4.7. Philosophy

4.8. Psychology

5. Social Science

5.1. Anthropology/Archaeology

5.2. Area Studies

5.3. Economics

5.4. Ethics

5.5. Geography

5.6. Political Science

5.7. Sociology

6. Applied Professional Science

6.1. Agriculture

6.2. Architecture

6.3. Business

6.4. Education

6.5. Engineering

6.6. Law

6.7. Management 


\subsection{Military Science}

The list of academic disciplines adds two more categories to traditional science:

7. Humanities

7.1. Creative Writing

7.2. History

7.3. Linguistics and Language

7.4. Literature

7.5. Logic

7.6. Metaphysics, Philosophy and Applied Philosophy

7.7. Religion

7.8. Semiotics and Semantics

8. Arts

8.1. Dance

8.2. Music

8.3. Painting

8.4. Performing Arts, Acting and Movies

8.5. Sculpture

8.6. Skilled Design and Crafts

8.7. Visual Arts, Movies, New Media (Video-computer Art)

These are the academic disciplines that are normally supported by most, but not all of western higher education. Most of these disciplines were developed, revised, and/or formalized in the 19th and 20th centuries. There is no overall consensus on how academic disciplines should be classified; every educational and science institution is free to adapt them to their own unique conditions.

The current developments in both science and academia are becoming heavily influenced by inter and transdiscipline approaches to exploration and development. This tendency will undoubtedly increase over the next ten years.

It is helpful to understand the difference in the similar but quite different terms used here: "multidisciplinary" refers to a non-integrative mixture of disciplines in that each discipline retains its methodologies and assumptions without change or development from other disciplines within the multidisciplinary relationship; "Interdisciplinary" is a field of study that crosses traditional boundaries believed academic disciplines or schools of thought, as new needs and professions have emerged; "Transdisciplinary" connotes a research strategy that crosses many disciplinary boundaries to create a holistic approach. In German speaking countries it refers to integration of diverse forms of research and includes specific methods for relating scientific knowledge and problem solving (Brand, Schaller, \& Völker, 2004). Given this new direction of science and academic development, it is suggested that the traditional categorization of disciplines include a new category to be included in the list above as: $\mathrm{C}$ inter/transdisciplinary studies. Within this new $\mathrm{C}$ category, it is suggested we include a science of information, plus some new multi-disciplines, such as cybernetics, history of science, neuro-psychology, artificial intelligence, social information science, information economics all which will contribute to this new trans-disciplinary field. This is illustrated in the graphic below. 


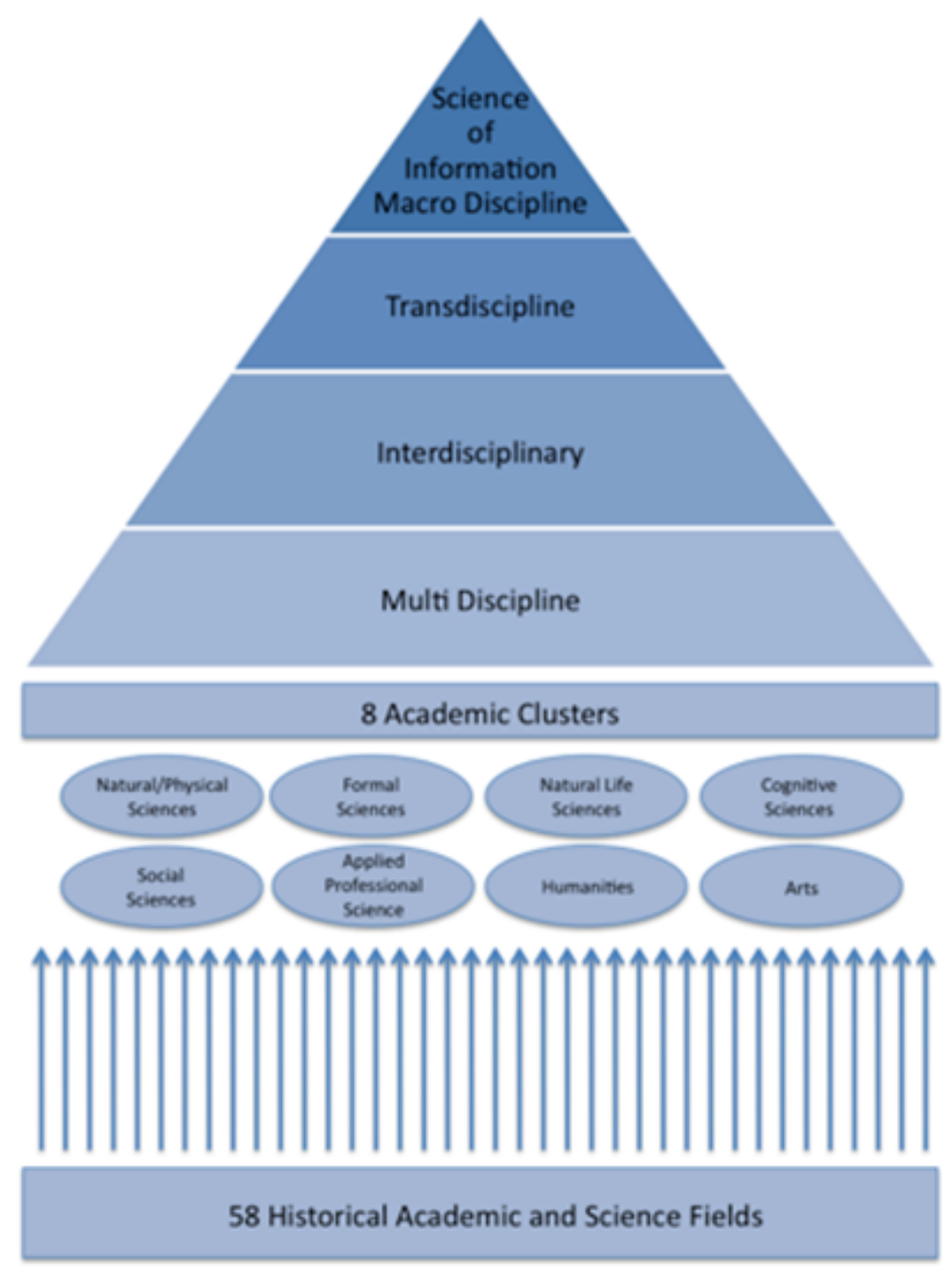

Figure 1: Chart of Contributing Information Studies: Toward Development of a New Science of Information

The new information transdisciplinary category is parallel to the physical, and social sciences/humanities divisions of academic subjects. What makes a difference is that this science of information discipline complements, assists and adds to the other disciplines by assembling and correlating all the information-related activities of the other disciplines. Eventually, we want the science of information to be developed well enough that colleges and universities will be willing to develop faculties and departments built around this subject.

In establishing the new evolved information categories, there should be some initial awareness that information is also a part of all elements, systems, and conditions. Therefore, it is also an integral part of the other individual disciplines and sciences. In studying the information phenomena, it is essential to look beyond the limitations of how humans use and perceive information, or even how other living organisms use information. It is proposed that information is a continuously evolving process, that exists in some simple to complex form in every stage of development, across all science and academia domains, as well as being a significant part of everything that exists. Information is a trigger mechanism, emphasis and nutrient, not only for information activities itself but 
also, all physical, and biological elements, systems and activities. This infers that we need to seriously study the possibility that there is a reality beyond the traditional understanding of the "physical universe."

In Paul Davies and Niels Henrik Gregersen's Book "Information and the Nature of Reality" (2010), Chapters 4, 5, and 6 Paul Davies, Seth Lloyd, and Henry Pierce Strapp challenge some widely held assumptions about physical reality. Davies asks what happens if we do not assume that the mathematical relations of the so-called laws of nature are the most basic level of description, but rather if information is regarded as the foundation on which physical reality is constructed. Davies suggests that instead of taking mathematics to be primary, followed by physics and then information, the picture should be inverted in our explanatory scheme, so that we find the conceptual hierarchy: information $\rightarrow$ laws of physics $\rightarrow$ matter. Lloyd's view of the computational nature of the universe develops this understanding by treating quantum events as 'quantum bits', or qubits, whereby the universe 'registers itself'. Lloyd approaches this subject from the viewpoint of quantum information science, which sets as a major goal the construction of a quantum computer - a device that can process information at the quantum level, thereby achieving a spectacular increase in computational power. The secret of a quantum computer lies with the exploitation of genuine quantum phenomena that have no analogues in classical physics, such as superposition, interference, and entanglement. Quantum computation is an intensely practical programme of research, but Lloyd uses the concept of quantum information science as the basis for an entire worldview, declaring that the universe as a whole is a gigantic quantum computer. In other words, nature processes quantum information whenever a physical system evolves.

It may be helpful to see chapter 2 of the Oxford University Press book "Every Thing Must Go: Metaphysics Naturalized" by James Ladyman, Don Ross, David Spurrett, and John Collier (2007). The Science of Information should take a lead in developing information as a new unique field, different, but in partnership with physical reality. This is a functional perspective, not a statement of philosophy or cosmology.

I suggest those actively engaged in this new field begin a current discussion with their associates, to derive specific activities and steps that are needed to assist in integrating this evolving discipline into a new science/academic category. It is suggested that the new science of information category be designated within a new category C "multi-disciplines" and be number 9 on the prior list. Moreover, we as colleagues should seriously study, suggest and share with each other how this new field could now actively be included in both the scientific and academic communities. The study of Information as a Science could very well become the most revealing and applicable scientific development of the Twenty-First Century.

\section{Antecedents of this Approach}

It may be helpful to understand how this particular concept of Information, as a Science, has evolved over my 70 years of work experience with the commercial, industrial, governmental and academic world, since it somewhat parallels the development of this evolving concept. In 1939 as a youth, I started working for a newspaper in California, which included working both the rear printing activity and then moving to the front publishing activity during my apprenticeship. In 1954, after completing a series of advanced courses on communications, information systems, and technology, I was tasked to start editing and publishing an internal federal government newsletter on information, and technical cultural developments. In 1955, I was appointed to a small team of system engineers, scientists and developers, who were tasked by the U.S. Government Advanced Research Projects Agency (ARPA) to look at newer ways to conceive, develop and use technical organizational information. A few of the recommendations of that research team have continued to influence information research and development, and included: (1) focusing of funding on digital rather than analog compilers, (2) develop, and correlate technical information, where possible, in integrated, outputs and display systems, rather than for a singular purpose. (3) Move the handling and processing of information from the existing batch processing, to a more interactive real time process (4) ensure that more members of the using organizations be involved in information related 
activities, rather than just relying on outside assistance and (5) move beyond traditional science and engineering technical specializations, and use a multidisciplinary conceptual, and planning approach, for all information system, application and developments.

In 1962, at University of California in Los Angeles (UCLA) a group of fellow lecturers and I prepared the second graduate seminar on Information Display Systems. We suggested to the Dean of Engineering that it would be preferable to approach technical information from a multi-discipline and user point of view, and add to the traditional scientific and engineering approaches, the techniques and perspectives of human factors, social sciences, arts, and humanities. In addition, we suggested the field of information processing and display were in essence, part of a larger interdisciplinary study and practice of information studies. The Dean agreed, and he adopted this new approach.

In 1970, Klaus Otten at National Cash Register Company, in Dayton, Ohio, and Anthony Debons, at Information Science Department, University of Dayton (who I later worked with) published a paper in the Journal of American Society for Information Science, entitled "Towards a Metascience of Information," (Journal of the American Society for Information Science, JanuaryFebruary 1970, Page 89), in which they summarized contemporary developments in the field. This had considerable influence on development of the emerging field of information science. Much of this paper is still relevant at the beginning of the 21 st century.

Further expansion of a unified study and development of information was presented during the first and second international conferences of the FIS (Foundations of Information Science), which took place in Madrid in 1994, and Vienna in 1996. Wolfgang Hofkirchner summarized and expanded those concepts in his edited volume "The Quest for a Unified Theory of Information" (1999).

In 1989, the American Physicist John Wheeler delivered a famous lecture entitled "it from bit", which stated in part "all things physical are information - theoretic in origin - and that, this is a participatory universe". Hans Christian von Baeyer, in his book "Information: the New Language of Science" (2003), and later Luciano Floridi, an information philosopher at Oxford University, in his book, "Information: A Very Short Introduction" (2010), indicated that the study of information should include the conceptual addition of physical science's contributions to the traditional Information Science studies.

These are the experiences that contributed to my suggesting that, all of these developments have led to a new, evolved "Science of Information."

\section{The Challenge Before Us Today}

In 2010, there is enough research, discussion, and interest in this subject to now begin the process of developing a new Science of Information, as a formal discipline, and academic specialty.

The new Science of Information must now be assembled by combining the work from multiple disciplines regarding information as a science; this then should assist in opening up expanded perspectives and opportunities within the traditional contributing discipline, as well as the new Science of Information. It should continue to recognize, honor, and assist, the traditional disciplines that have contributed to its beginning. It is important to continue to expand more deeply each of these contributing disciplines' contributions to all knowledge. It is not a purpose here to replace these traditional disciplines, but rather to create a new combined meta-science, to stand with and alongside them, in order to collaborate and enhance their activities. It is important to understand this meta-science would enhance what all disciplines and areas of scientific and academic research can contribute, by adding new perspectives, resources and insights. It should be a driving force in actively moving our old materially focused cultures toward a newer more responsive and enlightened information culture. It should take a leadership role in helping Science reintegrate its views via multi- and transdiscipline approaches toward a more holistic perspective of Science in our more contemporary society. 
All living species, systems and academic developments need to go through adaptation and transition periods in order to meet new developments, changing conditions, and challenges. So should the new study of information. We need to step out beyond where we have been up to now, and into a newer evolved combined macro challenge. The emergence of the field of "Life Science," has shown that the development of a new categorization of science and academia can be accomplished. It is important in forming the science of information, that we are aware, and cautious of, the influence of historical, cultural, political, economic and academic self interests. These wish to maintain the status quo, drain off focus, and shift resources so as to diminish the open, unified, cooperative, participation that will be needed to form this new field.

What we need most is personal commitment and action by each individual involved in some aspect of the disciplines involved in this new macro science, as well as the participants of this 2010 conference in Beijing. It is now up to all of you, the activists, to assist in moving this science of information into a viable field of scientific and academic research and study. It is possible that all our combined efforts can help not only establish a valuable new field of study and research, but also, to assist in understanding, adapting to civilization's increased rate of change, better offer perceptual understandings, and reduce misunderstanding of universal information principles, misuse of information perspectives, and then advance our capabilities to achieve other, yet currently unknown benefits for humanity.

\section{References}

Brand, F., Schaller, F., \& Völker H. (Eds.). (2004). Transdisziplinarität: Bestandsaufnahme und Perspektiven: Beiträge zur THESIS-Arbeitstagung im Oktober 2003 in Göttingen. Göttingen: Universitätsverlag Göttingen.

Davies, P., \& Gregersen, N. H. (2010). Information and the Nature of Reality: From Physics to Metapysics. Cambridge, UK: Cambridge University Press.

Floridi, L. (2010). Information: a very short introduction. Oxford, UK; New York, NY: Oxford University Press.

Hofkirchner, W. (Ed.). (1999). The Quest for a Unified Theory of Information. Amsterdam, NL: Gordon and Breach Publishers.

Ladyman, J., Ross, D., Spurrett, D., \& Collier, J., (2007). Every Thing Must Go: Metaphysics Naturalized. Oxford, UK; New York, NY: Oxford University Press.

Otten, K., \& Debons, A. (1970). Towards a Metascience of Information: Informatology. Journal of the American Society for Information Science 21(1), 89.

Schrödinger, E. (1992). What is life? With Mind and Matter and Autobiographical Sketches. Cambridge, UK: Cambridge University Press.

von Baeyer, H.C. (2003). Information: the New Language of Science. London, UK: Weidenfeld \& Nicolsen.

Wheeler, J. (1990). Information, Physics, Quantum: The Search for Links. In W. H. Zurek (Ed.), Complexity, Entropy, and the Physics of Information. Redwood City, CA: Addison-Wesley.

\section{About the Author}

Dail Dewitt Doucette

Dail Doucette, a scientist and systems designer, works in the area of interoperability, interconnection of Information Science and Technology issues between different academic, federal government departments and research disciplines. He has taught advanced information theories and display systems for the Engineering School at the University of California at Los Angeles, the American University Center for Technology (Washington, D.C.), and the NATO Science division (Brussels, Belgium). He was guest lecturer on the information aspects of cultural anthropology at the University of Kyoto (Japan), the American University in Beirut, the University of Southern California (USC), Cambridge University (United Kingdom), and the Fulbright Scholarship recipients orientation program for west coast U.S. universities. He assisted in formation of a joint Masters and Ph.D. program between USC and the University of Teheran (Iran). He was also Executive Vice President of ComFac, a commercial telecommunications corporation. He is a long-time active member of the American Association for the Advancement of Science, "Section T" (information, computing, and communications). He was an NGO delegate to the UNESCO World Summit on the Information Society in Geneva, Switzerland in 2003, and the second session in Tunis, Tunisia, in 2005. He was a member of the committee that founded the Society of Information Display, helped start the World Future Association, and is assisting in establishing the Science of Information Institute. 